\title{
EMG Analysis on Gyro-Roller Rehabilitation Device for Stroke Patients
}

\author{
Tulakan Ruangrong, Panrasee Ritthipravat* \\ Department of Biomedical Engineering, Mahidol University, Nakhon Pathom, Thailand. \\ * Corresponding author. Tel.: +66971917879; email: panrasee.rit@mahidol.ac.th. \\ Manuscript submitted January 13, 2017; accepted March 15, 2017. \\ doi: 10.17706/ijbbb.2017.7.3.153-161
}

\begin{abstract}
This paper presents a novel rehabilitation system, called "Gyro-roller", for stroke patients. The system utilizes gyroscopic effect and virtual reality technology to regain physical strength and functions of individuals with upper extremity disabilities. Efficacy of the gyroscopic effect is investigated by electromyography analysis on deltoideus, triceps brachii, biceps brachii, extensor carpi ulnaris and flexor carpi radialis muscles of 10 healthy subjects. Results show that all muscles are exercised when the subjects control the proposed device in any direction. The gyroscopic effect helps increasing muscle activities and can be adjusted to fit with individuals. An example of rehabilitation game is shown in this paper. The game was assessed using System Usability Scale (SUS) by physical and occupational therapists. The results showed that average percentile rank is of 73 which represents the game is more likely to be used in practice.
\end{abstract}

Key words: Gyro-roller, rehabilitation, EMG.

\section{Introduction}

Cardiovascular disease is one of the major causes of death among non-communicable diseases worldwide [1]. Stroke, a type of cardiovascular disease, mainly affects motor and cognitive functions of patients. Severity of the stroke depends on the characteristics and extend of damage to the brain. Nowadays, the number of stroke survivors has increased considerably. In Thailand, cerebrovascular accident is the third leading cause of death among non-communicable diseases reported in 2012. Each year, approximately 20,000 patients die from 200,000 new stroke patients [2], [3]. The impairments of stroke survivor can be divided into two major categories, upper and lower extremity disabilities. Normally, patients with lower extremity dysfunction can still walk but they may have an abnormal gait pattern [4]. Upper extremity dysfunction demands longer recovery time. It thus affects to patients' quality of life and requires continual rehabilitation [5].

Stroke rehabilitation is a lifelong process that involves repetitive tasks. Patients need to strictly perform both physical and occupational therapies in order to regain their functions. Task specific training and constraint-induced movement therapy (CIMT) are movement based therapeutic methods which have been commonly used in the last decade. CIMT concerns the effect called learned non-use which states that a stroke patient tends to avoid using the weakened limb even though it can still be moved slightly. CIMT is accomplished by constraining the less affected side of the limb and forcing paretic side to perform the task. Nevertheless, the patient often feels tired, bored, and discouraged due to its repetitive nature. Eventually 
there is no improvement in the rehabilitation. Virtual reality and robotic technology have been introduced in the past decade in order to address these shortcomings [6], [7].

Robotic device is normally used as a tool in rehabilitation because it can operate repeatedly with great consistency. In general, robotic system is usually integrated with virtual reality (VR) technology including interactive video gaming [8]. VR is a computer-based technology that creates virtual world and allows users to have realistic experiences through their perception [6]. It is one of the promising approaches in rehabilitation for stroke survivors in which patients can play computer games during the training. Currently, there are several robotic devices with VR technology. Robot assisted gait training (RAGT) facilitates gait recovery [4]. Exoskeleton for arm rehabilitation focuses on upper extremity training [9]. These systems received good feedback from the users.

From previous study [10], it was concluded that therapies should be done by combining multiple approaches rather than applying only single approach. Alternative rehabilitation devices are thus important in which patients can use various devices in training in order to make them feel more pleasurable. In this paper, a rehabilitation device, called Gyro-roller, has been introduced. The initial development began in 2012 [11] as seen in Fig. 1. Gyro-roller is developed based on the gyroscope precession principle. The device has a spherical shape with two handles and a rectangular base. The base has a motor for driving a wheel inside the Gyro-roller. User moves the Gyro-roller to play the computer games during rehabilitation. The counter torque or the gyroscopic effect, is generated when user controls the device. This effect is used for strengthening muscles of a user. However, the previous versions of Gyro-roller can shortly maintain the gyroscopic effect because the spinning wheel is not constantly driven. New version of Gyro-roller is thus developed [12], [13] and presented in this paper. To show efficacy of the gyroscopic effect in rehabilitation, electromyography (EMG) signals are measured. This paper aims to investigate activation of EMG signals in five muscle groups while controlling the Gyro-roller. EMG activation pattern can be used as a guideline for game design. An example of rehabilitation game is also provided herein with the usability testing.

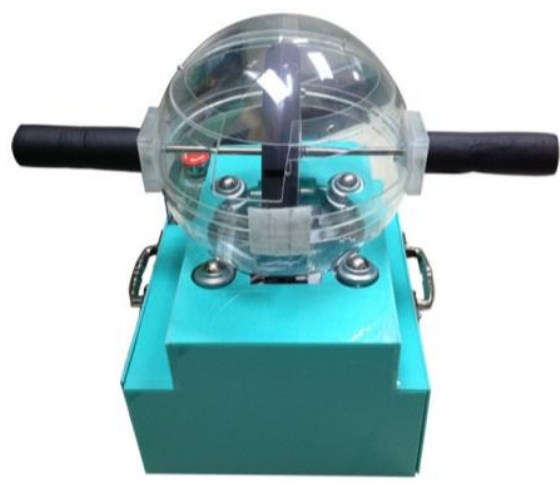

Fig. 1. The first version of Gyro-roller.

\section{Materials and Methods}

\subsection{Gyro-Roller System}

Gyro-roller is a rehabilitation device that uses gyroscopic effect to strengthen muscles in upper extremity part of stroke patients. It has a spherical shape and two handles. Gyro-roller is connected with a computer which displays a virtual environment to a user. Inside the Gyro-roller, it has a wheel which is driven by a DC motor as shown in Fig. 2. Speed of the motor can be varied. A user interacts with a virtual environment by controlling the Gyro-roller. It supports two degrees of freedom, i.e., roll and yaw directions as presented in Fig. 3. The direction of counter torque is perpendicular to the axis of user input, while its magnitude varies 
with the spinning velocity.

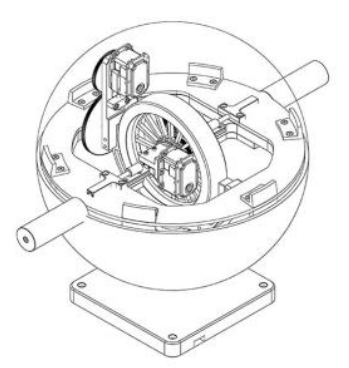

Fig. 2. Gyro-roller device.

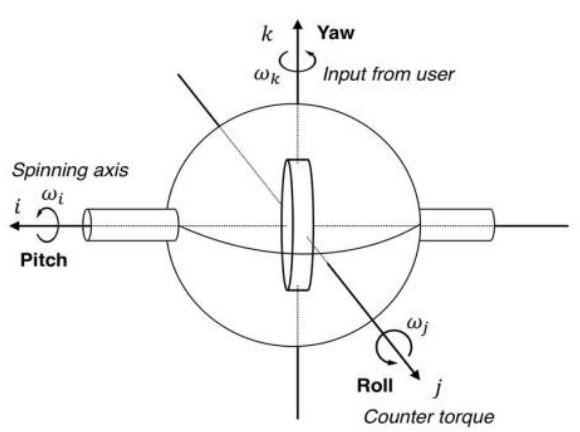

Fig. 3. Gyro-roller coordinations.

\subsection{Gyroscopic Effect}

Gyroscopic effect represents the counter torque generated when user applies torque to move the Gyro-roller. The moment of inertia of the wheel $(I)$ inside the Gyro-roller can be determined from its mass $(m)$, inner and outer radii $\left(R_{1}\right.$ and $\left.R_{2}\right)$

$$
I=\frac{1}{2} m\left(R_{1}^{2}+R_{2}^{2}\right)
$$

Angular momentum of the wheel can be computed from the product of $I$ and the angular velocity, $\omega_{i}$, of the wheel as follows

$$
L=I \omega_{i}
$$

Counter torque $(\tau)$ is then determined from rate of change of angular momentum

$$
\tau=\frac{d L}{d t}=I \omega_{i} \cdot \omega
$$

where $\omega$ represents angular velocity arisen when user controls the Gyro-roller in either roll $\left(\omega_{j}\right)$ or yaw $\left(\omega_{k}\right)$ orientations.

\subsection{Example of Rehabilitation Game}

One of the rehabilitation games called GDrive is a coin-collecting game. The GDrive game is developed based on Unity в Game Engine. User has to move virtual motorcycle by controlling Gyro-Roller to the left or right for collecting the coin falling down from top of the screen. There are three lanes for moving the 
motorcycle as seen in Fig. 4. The coin is spawned one by one with the adjustable interval. The motorcycle is displayed on the bottom of the screen. Score and time counter are shown on the top-left and top-right of the screen respectively. The game has three difficulty levels, easy, medium and hard which control the interval of coin spawning (every 3, 2, and 1 seconds correspondingly). The coin spawning positions can be set to fit with individual need. User can set whether to control the Gyro-roller in either yaw or roll rotations.

To evaluate the designed game, the System Usability Scale (SUS) is used. It is a reliable tool for assessing the usability of a system whether it should be used in practice. It is technology independent and generally used as industry standard. It has been used to evaluate hardware, software and websites [14]. There are ten questions as seen in Table I, with five evaluation scores from strongly agree (5) to strongly disagree (1). The items in odd-numbered indexes are positive questions and even-numbered indexes are negative questions. In this paper, five therapists (three physical therapists and two occupational therapists) were asked to answer the SUS questionnaire after testing the GDrive game.

Table 1. System Usability Scale Scores from Physical and Occupational Therapists

\begin{tabular}{|c|c|c|c|c|c|c|}
\hline Question & PT1 & PT2 & PT3 & OT1 & OT2 & Avg. \\
\hline 1. I think that I would like to use this game frequently. & 4 & 3 & 2 & 4 & 2 & 3 \\
\hline 2. I found the game unnecessarily complex. & 1 & 1 & 4 & 1 & 2 & 1.8 \\
\hline 3. I thought the game was easy to use. & 3 & 3 & 1 & 4 & 2 & 2.6 \\
\hline $\begin{array}{l}\text { 4. I think that I would need the support of a technical person to be able to use } \\
\text { this game. }\end{array}$ & 1 & 1 & 1 & 1 & 1 & 1 \\
\hline 5. I found the various functions in this game were well integrated. & 4 & 4 & 5 & 4 & 2 & 3.8 \\
\hline 6. I thought there was too much inconsistency in this game. & 1 & 1 & 1 & 1 & 2 & 1.2 \\
\hline $\begin{array}{l}\text { 7. I would imagine that most people would learn to play this game very } \\
\text { quickly. }\end{array}$ & 4 & 4 & 2 & 5 & 2 & 3.4 \\
\hline 8. I found the game very cumbersome to play. & 1 & 1 & 4 & 1 & 2 & 1.8 \\
\hline 9. I felt very confident using the game. & 4 & 5 & 3 & 4 & 2 & 3.6 \\
\hline 10. I needed to learn a lot of things before I could get going with this game. & 1 & 1 & 3 & 1 & 1 & 1.4 \\
\hline Percentile Rank & 85 & 85 & 50 & 90 & 55 & 73 \\
\hline
\end{tabular}

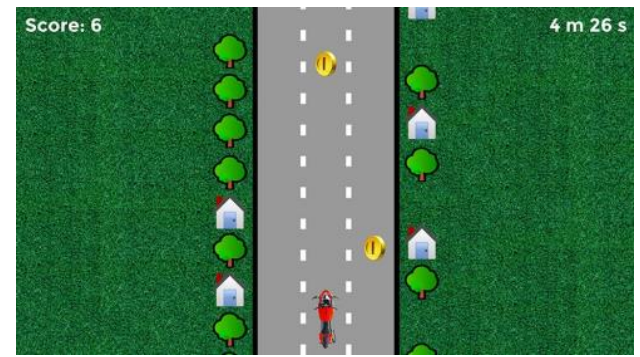

Fig. 4. A prototype game, GDrive.

\section{Experiment}

The experiment aims to investigate the effect of counter torque to muscle activation. Ten healthy subjects (five males and five females) who are graduated students from Faculty of Engineering, Mahidol University, were recruited. All participants could move their upper extremities normally. No one had been injured or had muscle fatigue before the experiment. Electromyography (EMG) signals were measured by wireless surface electrodes as seen in Fig. 5. Instructions for using the Gyro-roller were introduced to each subject. The subject sat in front of the Gyro-roller and grabbed the handles in order to control the virtual objects displayed on the computer screen. In the experiment, a simple game was developed by the use of Unity $\mathrm{R}$ Game Engine. It consists of two objects, i.e., a green ball and a blue bar as presented in Fig. 6. The bar is set to move side to side with a constant speed. The subject must control the Gyro-roller to move the ball to 
overlap the bar. Once the ball is aligned with bar, its color is changed from red to green. Each subject is trained to play the game before the experiment. By doing this, users can control the Gyro-roller with the similar speed. There are eight steps for testing. They are presented as follows.

1) Relax.

2) Grabbing on the handles.

3) Moving only in yaw direction with no gyroscopic effect (the wheel inside the Gyro-roller is not spun).

4) Moving only in yaw direction with the gyroscopic effect (the wheel inside the Gyro-roller is spun).

5) Moving only in roll direction with no gyroscopic effect.

6) Moving only in roll direction with the gyroscopic effect.

7) Moving only in roll and yaw directions with no gyroscopic effect.

8) Moving only in roll and yaw directions with the gyroscopic effect.

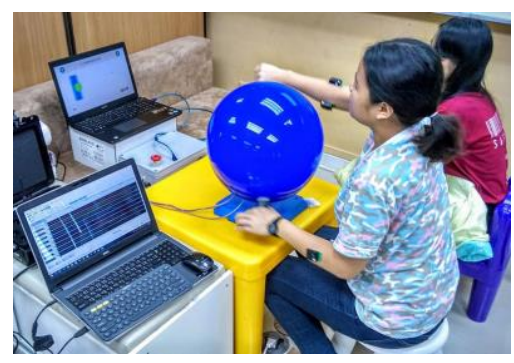

Fig. 5. EMG collection environment setup.

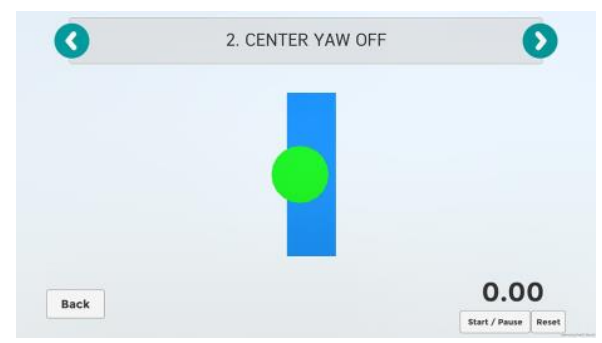

Fig. 6. A simple control game for EMG measurement.

Each subject was asked to perform each step for 60 seconds. Delsys Trigno ${ }^{\text {TM }}$ Wireless Systems and Smart Sensors (DelSys Incorporated, Boston, MA, USA) were used to record EMGs from both sides of deltoideus, triceps brachii, biceps brachii, extensor carpi ulnaris and flexor carpi radialis muscles (10 channels in total). The electrodes were placed by the same researcher after cleaning subjects' skin with alcohol solution. The EMG signals were acquired by EMGworks R Acquisition with a sampling frequency of $1926 \mathrm{~Hz}$.

Rehabilitation games should be designed to serve patients which have different age groups, sex, educational levels and muscle impairment. Prior to designing the games, electromyography (EMG) signals are measured while controlling the Gyro-roller in order to ensure that certain muscles are activated.

After measuring EMG signals from all subjects. Bandpass filter with the range of $20 \mathrm{~Hz}$ to $500 \mathrm{~Hz}$ were applied in order to remove noises, artifacts including baseline shifts [15]. Root mean square (RMS) of the filtered EMGs [16] were then calculated as follows:

$$
R M S=\sqrt{\frac{1}{N} \sum_{i=1}^{N} x_{i}^{2}},
$$

where $N$ is a window length of the filter. $x_{i}$ represents the $i^{\text {th }}$ sampled EMG signal. Overall processing steps 
can be seen in Fig. 7.

\section{Results}

\subsection{EMG Results}

Average RMS values of all subjects were computed and presented in Fig. 8. In this figure, there are three sub-figures which represent user's movement patterns, i.e., roll, yaw, and both directions. White and gray bars represent inactive and active gyroscopic effects respectively. Student's tablet-test was applied to compare the differences between active and inactive gyroscopic effect conditions for each movement. The derived $p$-values are shown in Table 2. The values with significant level lower than 0.05 are labeled as bold text.

Table 2. P-Value From Student's t-test of EMG Signals

\begin{tabular}{|c|c|c|c|c|c|c|c|c|c|c|}
\hline \multirow{2}{*}{ Tasks } & \multicolumn{5}{|c|}{ Left } & \multicolumn{5}{|c|}{ Right } \\
\hline & Biceps & Deltoid & Extensor & Flexor & Triceps & Biceps & Deltoid & Extensor & Flexor & Triceps \\
\hline Yaw & 0.003 & 0.190 & 0.013 & 0.049 & 0.003 & 0.013 & 0.297 & 0.001 & 0.022 & 0.002 \\
\hline Roll & 0.269 & 0.214 & 0.000 & 0.092 & 0.002 & 0.714 & 0.096 & 0.001 & 0.005 & 0.037 \\
\hline Both & 0.043 & 0.017 & 0.080 & 0.144 & 0.041 & 0.117 & 0.004 & 0.005 & 0.264 & 0.049 \\
\hline
\end{tabular}

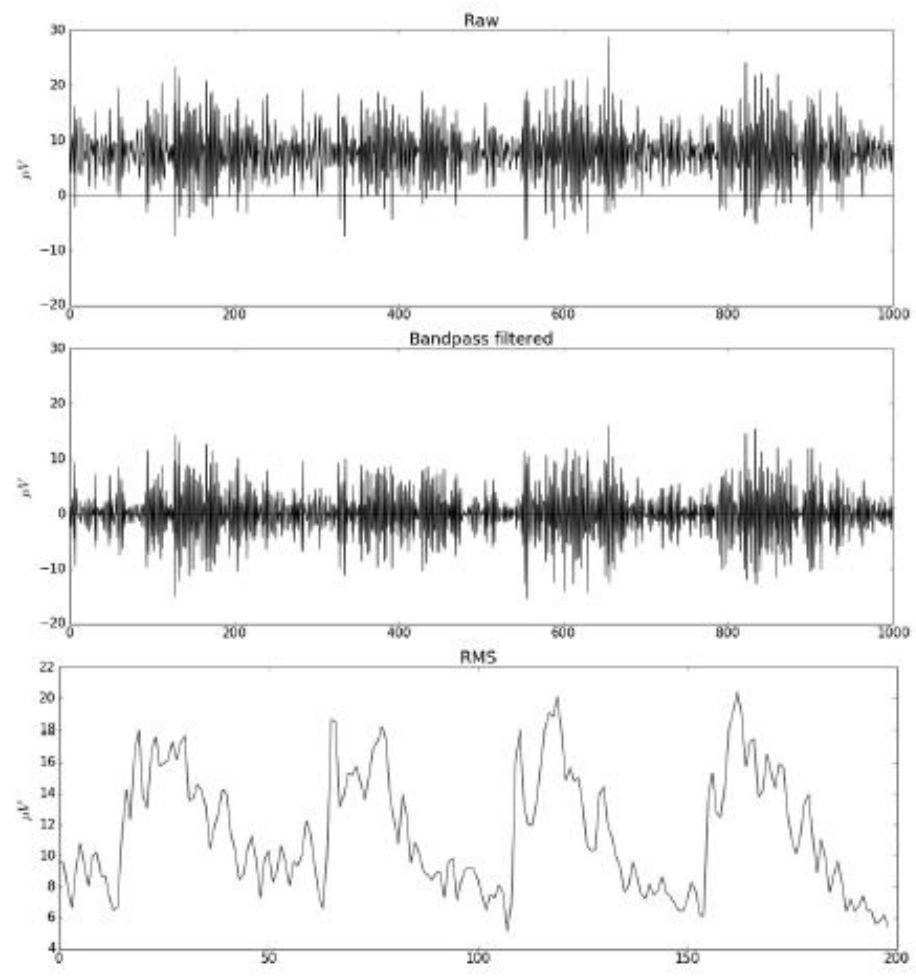

Fig. 7. EMG processing steps.

\subsection{Designed Rehabilitation Game with Usability Testing}

The System Usability Scale are computed based on the method described in [14]. Firstly, the scores of the odd-numbered indexes in the questionnaire are subtracted by one. Secondly, those of the even-numbered indexes are subtracted from five. These procedures normalize each score to be in between 0 to 4 . All normalized values are then summed up and scaled by a factor of 2.5 . The scaled value is presented as the percentile rank. The evaluation scores and percentile rank from each therapist are shown in Table 1. 

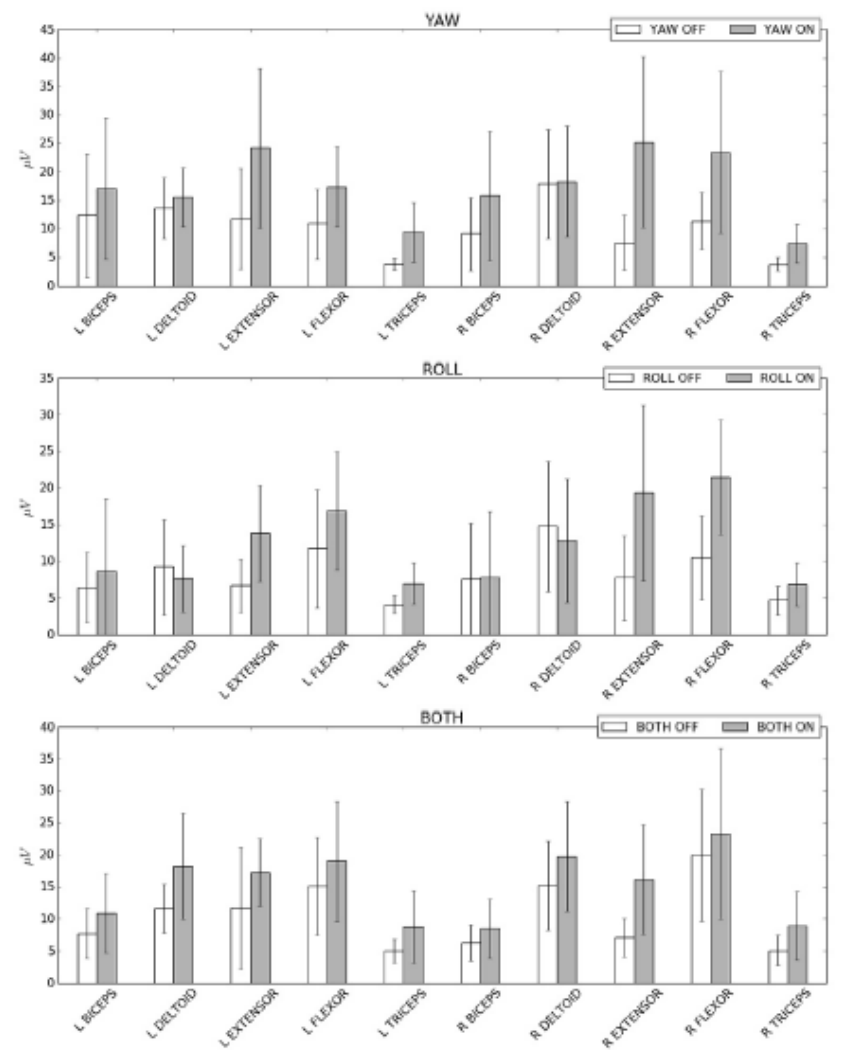

Fig. 8. Means and standard deviations of RMS values in each movement.

\section{Discussion}

From the experimental results, all muscles are activated when users control the Gyro-roller. When the wheel inside the Gyro-roller is spun, most of the muscles have higher average RMS values compared to that of no-spinning wheel condition. Subjects have to overcome the counter torque in order to move the Gyro-roller. In clinics, this torque can be adjusted by changing speed of the spinning wheel. It can be set to fit with each patient. This shows an advantage of Gyro-roller system. For controlling the Gyro-roller only in roll direction, the counter torque acts in a supportive manner to the movement of deltoideus. The average RMS values in an inactive gyroscopic condition is thus higher than that of the active one. However, the student's $t$-test show that there is no significant difference (p-values of deltoideus are 0.214 and 0.096) as seen in Table 2.

Triceps are more activated when gyroscopic effect is active in any movement direction. Activity of Extensor carpi shows similar trend with that of triceps, except for the left hand side. It may result from all subjects being right-handed and tending to use it more than the left side. Because the device is placed on a table, moving in roll direction is thus constrained.

From the above study, Gyro-roller can generate gyroscopic effect to strengthen all muscles, particularly in triceps brachii, biceps brachii, extensor carpi ulnaris and flexor carpi radialis. This EMG analysis can be used as a guideline for selecting appropriate movements for each patient.

For the designed rehabilitation game, the results of System Usability Scale show that most of the therapists are relatively satisfied with the game. The average percentile rank is 73 which demonstrates the likeliness of the system to be used in practice. However, two of the therapists experienced cybersickness which may occur in patients. They suggested that some parts of the game background, such as tree and house, should not be moved when the motorcycle is driven. 


\section{Conclusion}

A novel rehabilitation device, called "Gyro-roller" is presented in this paper. It applied gyroscopic effect to strengthen muscles in upper extremity part. The efficacy of this gyroscopic effect is validated by EMG analysis of 10 healthy subjects. An example of rehabilitation game is also shown in this paper with usability testing results. The percentile rank of SUS is 73 which is above average. Therefore, physical and occupational therapists can use Gyro-roller as an alternative rehabilitation device. In the future work, more rehabilitation games should be designed and tested in clinics.

\section{Acknowledgment}

This research was funded by Mahidol University Research Grant. The authors would like to thank therapists from Saraphi Hospital, Chiang Mai, Thailand for their help.

\section{References}

[1] WHO. (2013). Types of cardiovascular disease. Retrieved from the website: http://www.who.int/ cardiovascular diseases/en/cvd atlas 01 types.pdf

[2] Thai Bureau of Policy and Strategy. (2013). Number of deaths and death rates per 100,000 populations by leading causes of death. Retrieved from the website: http://bps.ops.moph.go.th/Healthinformation/2.3.6 53.pdf

[3] Thai Bureau of Non Communicable Disease. (2013). Number of vitalities caused by cerebrovascular accident. Retrieved from the http:// thaincd.com/information-statistic/non-communicable-disease-data.php

[4] Alex, L. E., Banala, S. K., Kim, S. H., Agrawal, S. K., \& Scholz, J. P. (2009). Robot assisted gait training with active leg exoskeleton (ALEX). IEEE Transactions on Neural Systems and Rehabilitation Engineering, 17(1), 2-8.

[5] Williams, L. S., Weinberger, M., Harris, L. E., Clark, D. O., \& Biller, J. (1999). Development of a stroke-specific quality of life scale. Stroke; A Journal of Cerebral Circulation, 30(7), 1362-1369.

[6] Arya, K. N., Pandian, S., Verma, R., \& Garg, R. K. (2011). Movement therapy induced neural reorganization and motor recovery in stroke: A review. Journal of Bodywork and Movement Therapies, 15(4), 528-537.

[7] Brewer, L., Horgan, F., Hickey, A., \& Williams, D. (2013). Stroke rehabilitation: recent advances and future therapies. Qjm, 106(1), 11-25.

[8] Takeuchi, N., \& Izumi, S. I. (2013). Rehabilitation with poststroke motor recovery: A review with a focus on neural plasticity. Stroke Research and Treatment.

[9] Frisoli, A., Borelli, L., Montagner, A., Marcheschi, S., Procopio, C., Salsedo, F., Bergamasco, M., Carboncini, M. C., Tolaini, M., \& Rossi, B. (2007). Arm rehabilitation with a robotic exoskeleleton in virtual reality. Proceedings of 2007 IEEE 10th International Conference on Rehabilitation Robotics, ICORR'07 (pp. 631-642).

[10] Cramer, S. C. (2008). Repairing the human brain after stroke. II. Restorative therapies. Annals of Neurology, 63(5), 549-560.

[11] Kulpavaropas, P., Makarananda, P., Rohitasathira, N., \& Ritthipravat, P. (2013). Gyro roller, the upper extremity rehabilitation system. Proceedings of Panyapiwat Academic Conference III. Nonthaburi, Thailand: Panyapiwat Institute of Management.

[12] Ritthipravat, P. (2014). Rehabilitation system for stroke patients: Upper extremity part. Research Report. Mahidol University.

[13] Ritthipravat, P., \& Rohitasathira, N. (2014). Gyro-roller: A rehabilitation device for stroke patients. Goal 
Medal Award in The 9th Taipei International Invention Show \& Technomart. Invention Intellectual Property Associations.

[14] Sauro, J. (2011) Measuring usability with the system usability scale (SUS). Retrieved from the website: http://www.measuringu.com/sus.php

[15] Konrad, P. (2005). The abc of emg. Retrieved from the website: http://demotu.org/ aulas/controle/ABCofEMG.pdf

[16] Phinyomark, A., Phukpattaranont, P., \& Limsakul, C. (2012). Feature reduction and selection for EMG signal classification. Expert Systems with Applications, 39(8), 7420-7431.

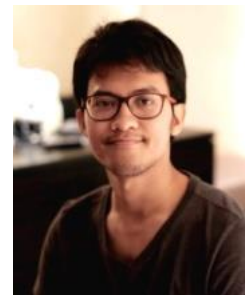

Tulakan Ruangrong received his B.Sc. in zoology from Kasetsart University, Thailand since 2011. He is currently pursuing M.Eng. in biomedical engineering at Mahidol University, Thailand. His research focuses on virtual reality based rehabilitation system.

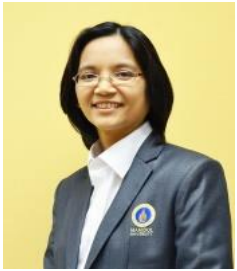

Panrasee Ritthipravat received the B.S. and D.Eng. in mechanical engineering, and the M.S. in modern manufacturing Management, King Mongkuts University of Technology Thonburi, Thailand. Her research interests include medical image processing, machine learning, intelligent control, robotics and assistive. 